\title{
Bloqueo auriculo-ventricular avanzado y bradicardia extrema como síntomas iniciales de Covid-19: Reporte de un caso
} Advanced atrioventricular block and extreme bradycardia as initial symptoms of Covid-19: A case report

Jorge Luis Vélez Páez, Mario Patricio Montalvo Villagomez, Estefanía Belén Irigoyen Mogro, Pedro Augusto Molina Vasquez

DOI. 10.21931/RB/2020.05.02.9

Resumen: Estamos viviendo la pandemia generado por el beta coronavirus SARS-CoV-2, sus manifestaciones clínicas son fundamentalmente respiratorias. Sin embargo, existen pacientes que podrían debutar con clínica distinta a la respiratoria, por ejemplo, gastrointestinal (diarrea); alteraciones sensitivas en el gusto y el olfato (anosmia, disgeusia) y cardiovasculares (arritmias, muerte súbita cardíaca, trastornos de la conducción), saliendo de esta manera de la presentación típica.

Los síntomas cardiovasculares y específicamente las alteraciones cardíacas, podrían ser causados por una mayor cantidad de receptores para la enzima convertidora de angiotensina 2 (ACE2), que es el sitio de unión del virus a las células humanas.

Se presenta un caso con diagnóstico confirmado de COVID -19, de una paciente adulta mayor, con comorbilidad cardiovascular, que debuta con arritmias y bloqueo cardíaco.

Palabras clave: COVID-19, SARS-CoV-2, arritmias cardíacas.

Abstract: We are experiencing the pandemic generated by the beta coronavirus SARS-CoV-2; its clinical manifestations are mainly respiratory. However, some patients could debut with clinical symptoms other than respiratory, for example, gastrointestinal (diarrhea); Sensory changes in taste and smell (anosmia, dysgeusia), and cardiovascular (arrhythmias, sudden cardiac death, conduction disorders), thus leaving the typical presentation.

Cardiovascular symptoms, and specifically cardiac disturbances, could be caused by an increased number of angiotensinconverting enzyme 2 (ACE2) receptors, which is the site of binding of the virus to human cells.

We present a case with a confirmed diagnosis of COVID -19, of an older adult patient with cardiovascular comorbidity, debuting with arrhythmias and heart block.

Key words: COVID-19, SARS-CoV-2, cardiac arrhythmias.

\section{Introducción}

Las manifestaciones clínicas del SARS-CoV-2, están dominadas por síntomas respiratorios, sin embargo, existe un número no despreciable de pacientes que podrían debutar con clínica primaria de daño cardiovascular, saliendo de esta manera de la presentación típica que es la sintomatología respiratoria ${ }^{1}$.

El SARS-CoV-2, así como el SARS-CoV, infectan a las células huésped por la unión de su proteína espiga a los receptores ACE2 (enzima convertidora de angiotensina 2), receptores estos que se encuentran predominantemente en pulmón y corazón. Estos receptores ACE2 tienen una importante función en el sistema cardiovascular e inmune, y se los ha asociado al desarrollo de hipertensión arterial y diabetes mellitus. El SARS-CoV-2 invade principalmente las células epiteliales alveolares, lo que conduce obviamente a compromiso respiratorio con la sintomatología respiratoria clásica. Estos síntomas son más graves en pacientes adultos mayores con enfermedad cardiovascular y comorbilidades preexistentes tales como: hipertensión arterial, enfermedad coronaria, insuficiencia cardíaca, diabetes ${ }^{2}$. Por tal razón no es de extrañar que en este grupo de pacientes exista una mayor proporción de muertes ${ }^{3}$.

El mecanismo de lesión aguda del miocardio podría responder a una mayor secreción de ACE2. De igual forma se ha planteado la posibilidad de una mayor expresión de receptores ACE2 en los pacientes que usan inhibidores del sistema renina-angiotensina-aldosterona, por lo que se podría considerar población de mayor riesgo, aseveración que aún no ha sido definida².

Otros mecanismos propuestos para la lesión miocárdica incluyen una tormenta de citoquinas desencadenada por una respuesta desbalanceada de células $T$ helper tipo 1 y tipo $2^{1,4}$ asociado además a disfunción respiratoria e hipoxemia, que provocan daño de las células miocárdicas.

Por lo tanto, en pacientes con SARS-CoV-2, es evidente que la incidencia de síntomas cardiovasculares no es despreciable y más bien diríamos frecuente, con desenlaces más severos y alta mortalidad por lo que deberemos ser más cuidadosos al momento del diagnóstico pues esto tendrá repercusiones no solo en la evolución, sino también en el incremento de contagios. Dato importante a tener en cuenta además es el daño cardíaco relacionado con los medicamentos durante el tratamiento, en particular el uso de medicamentos antivirales, cloroquina, hidroxicloroquina, los cuales pueden causar insuficiencia cardíaca, arritmia u otros trastornos cardiovasculares que deben ser monitoreados estrechamente ${ }^{5,6}$.

Se presenta un caso con diagnóstico confirmado de COVID -19, de una paciente adulta mayor, con comorbilidad cardiovascular, que debuta con arritmias y bloqueo cardiaco que precede a la clínica respiratoria.

\footnotetext{
${ }^{1}$ Universidad Central del Ecuador-Hospital Pablo Arturo Suárez

${ }^{2}$ Hospital Pablo Arturo Suárez.
} 


\section{Caso clínico}

Mujer de 81 años de edad, procedente de Quito, mestiza, con antecedente de hipertensión arterial en tratamiento con losartán 50 miligramos y carvedilol 12.5 miligramos. Presentó un cuadro clínico de síncope con una duración de un minuto, motivo por el cual es llevada a una unidad de salud donde se le realizan exámenes, evidenciándose en el electrocardiograma bloqueo auriculo-ventricular de tercer grado por lo que se indica colocación de marcapaso, sin embargo, los familiares solicitan alta voluntaria.

Presenta un nuevo episodio de síncope en el domicilio, es trasladada a emergencia, donde presentó parada cardiorespiratoria de 2 minutos con retorno a circulación espontánea. Valorada por Terapia Intensiva (UTI), se confirmó bloqueo A-V de tercer grado así como signos de bajo gasto decidiendo su ingreso.

En UTI se presentó consciente, bradicárdica con frecuencia cardíaca entre 30 a 40 latidos por minuto, que no genera hipotensión TAM: 90 - 100 mmHg, Respiratoriamente, 14 respiraciones por minuto, con cánula nasal 2 litros por minuto tiene Sat02: 89\%. Al examen físico, consciente, sin signos neurológicos, en corazón ruidos cardiacos arrítmicos, sin soplos; en pulmones, murmullo vesicular disminuido en bases.

En analítica como hallazgos relevantes se encuentra una troponina I elevada (0.33 VN: 0.00 a 0.01). Gasometría sin hipoxemia, ni hipercapnea. Dímero D elevado (5788 VN: 45-500), electrocardiograma mostró bloqueo auriculo-ventricular de tercer grado. (Figura 1)

Durante su hospitalización, la paciente presenta seis eventos más de parada cardiorespiratoria sin buena respuesta a marcapaso transcutáneo, atropina y aminas vasoactivas; 36 horas después de su ingreso a UTI presentó tos seca y cefalea, incrementó su trabajo respiratorio con taquipnea e hipoxemia gasométricamente; se solicitó radiografía de tórax que mostró infiltrado alveolointersticial bilateral difuso más cardiomegalia grado III en control radiográfico (Figura 2).

Teniendo en el deterioro clínico respiratorio, los hallazgos en la placa de tórax, el valor del dímero D muy elevado, la edad y comorbilidad, asociado al estado de epidemia por SARSCov2, se solicitó PCR el cuál es positivo.

La paciente es llevada a un sitio de aislamiento respiratorio, permanece hospitalizada, a los 8 días revierte el bloqueo de forma espontánea (sin apoyo de marcapaso). No requirió ventilación mecánica. Realiza hipoxemia súbita que lleva parada cardiorrespiratoria, la paciente fallece.

\section{Discusión}

El monitor del impacto cardiovascular de las infecciones virales ha sido la influenza. La miocarditis, el infarto agudo de miocardio y la exacerbación de la insuficiencia cardíaca, han sido reportadas durante epidemias históricas previas y la consecuencia común ha sido el aumento de la mortalidad ${ }^{7}$. Situaciones similares han sido descritas en brotes previos de coronavirus como el SARS y el MERS.

La edad avanzada, las enfermedades cardiovasculares preexistentes, la gravedad del impacto en la oxigenación, determinan resultados adversos ${ }^{8}$. La hipotensión, la taquicardia, la bradicardia, las arritmias e incluso la muerte cardíaca súbita son comunes en pacientes con SARS. Los cambios electrocardiográficos compatibles con lo descrito y la elevación de la troponina sugieren miocarditis viral, en la ecocardiografía suele hallarse insuficiencia diastólica ventricular izquierda subclínica, los que desarrollan insuficiencia sistólica y fracción de eyección reducida, tienen alta probabilidad de ingresar a ventilación mecánica9,10.

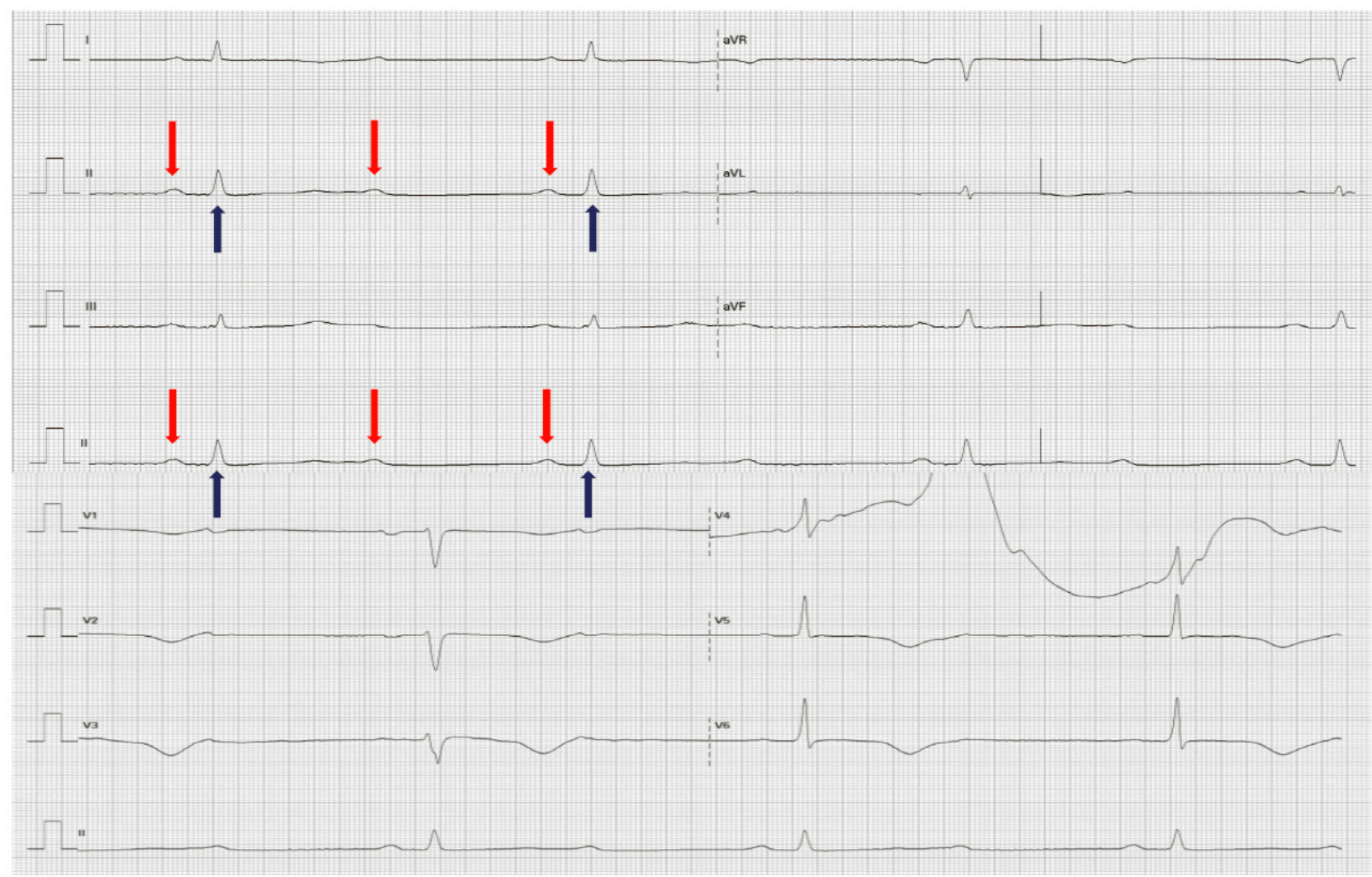

Figura 1. Electrocardiograma que demuestra bloqueo auriculo-ventricular de tercer grado. Flechas rojas: ritmo auricular, flechas azules: ritmo ventricular. Frecuencia cardiaca: 20 latidos por minuto. 


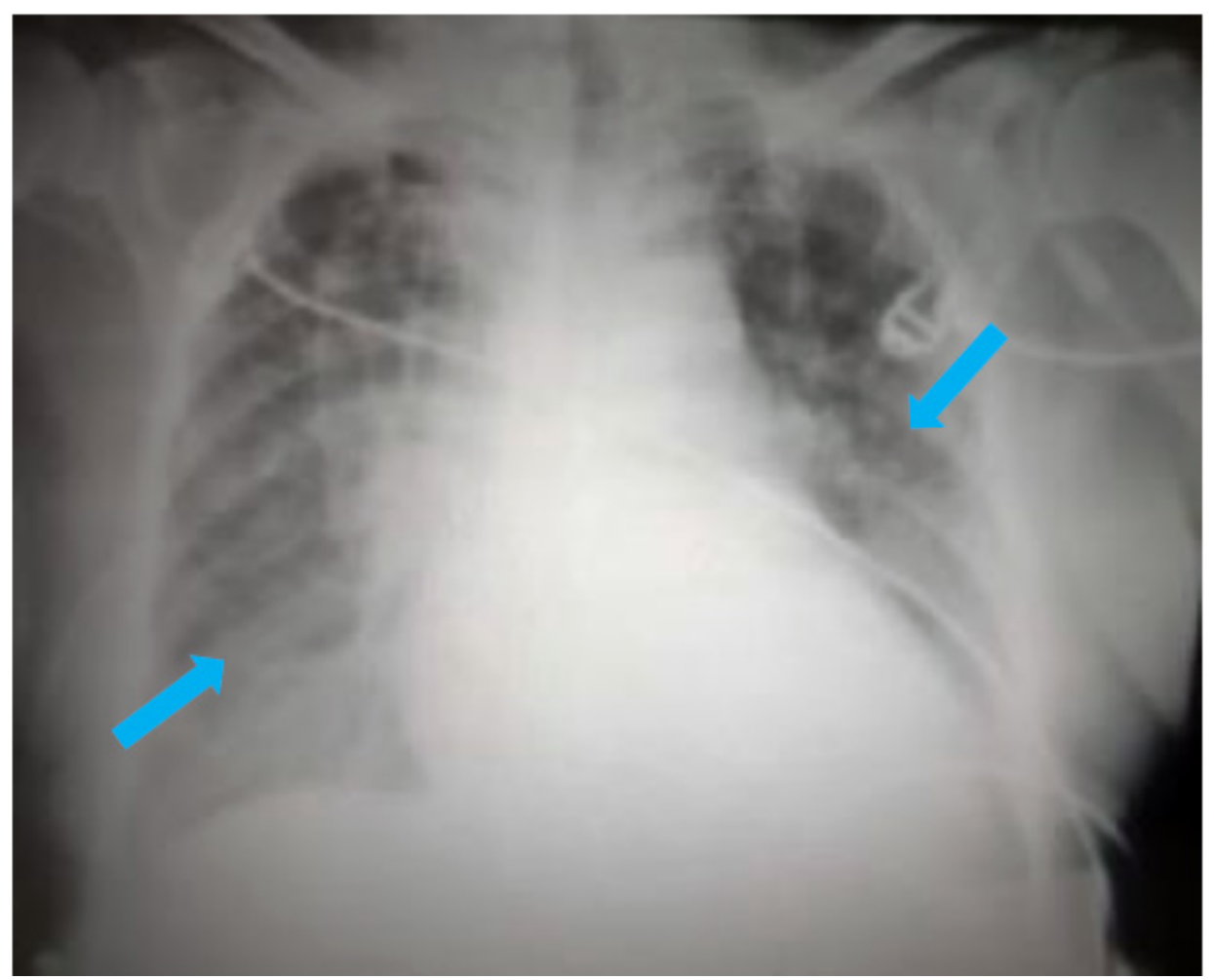

Figura 2. Radiografía antero-posterior de tórax. Flechas celestes indican infiltrado en parches bilateral difuso.

Las enfermedades virales, como toda infección sistémica generan aumento en la demanda metabólica y esto en pacientes con reserva cardíaca reducida por enfermedad cardiovascular crónica descompensa agudamente a éstos enfermos. La coexistencia de enfermedad coronaria e insuficiencia cardiaca expone a un riesgo particular de ruptura de la placa aterosclerótica secundaria a la inflamación sistémica, es por ello que el infarto agudo de miocardio es una complicación reportada.

EL SARS y el SARS-CoV-2 son beta-coronavirus que se unen a las células que expresan receptores para la enzima convertidora de angiotensina 2 (ACE2) ${ }^{11}$. La ACE 2 también se expresa en el corazón, permitiendo así que entren los coronavirus al sistema cardiovascular.

Los modelos murinos y las muestras de autopsias humanas demuestran que el SARS-CoV puede regular a la baja las vías de ACE2 miocárdicas y pulmonares, por lo tanto, mediando la inflamación del miocardio, el edema pulmonar y la insuficiencia respiratoria aguda.

En el caso reportado, se presenta un bloqueo auriculo-ventricular de tercer grado de instauración aguda, en una paciente de edad avanzada e hipertensa, que lleva a síncope cardíaco y eventos sucesivos de parada cardiorrespiratoria. Seguramente la etiología del bloqueo, sin síntomas respiratorios iniciales, se la debe atribuir en ésta paciente a causas isquémicas o farmacológicas, ya que la paciente tiene factores de riesgo cardiovascular, es tomadora de betabloqueantes y hubo elevación enzimática cardíaca. Sin embargo, lo agudo de la instauración del cuadro clínico en una paciente previamente asintomática, la refractariedad a marcapasos transcutáneo, atropina y aminas vasoactivas, y sobre todo que los síntomas cardíacos preceden en 36 horas a la clínica respiratoria y a una imagen pulmonar en radiografía de tórax con infiltración difusa bilateral, con PCR para SARS Cov -2 positivo; nos posiciona en un COVID -19 atípico; más aún cuando el bloqueo revierte espontáneamente, sin la necesidad de marcapaso.

Sobre este tema, hay amplio material reportado en expe- riencias epidémicas previas por otros coronavirus. En SARS, Yu et al. ${ }^{12}$ en 121 pacientes reportaron síntomas cardiovasculares como hipotensión, taquicardia, bradicardia, cardiomegalia y arritmias; Pan et al. ${ }^{13}$ en 15 pacientes describen muerte súbita cardíaca y Li et al. ${ }^{9}$ en 46 pacientes con ecocardiografía detectan insuficiencia diastólica subclínica sin fallo sistólico y que revirtió al superar la enfermedad. En MERS, Alhogbanii ${ }^{14}$ en un paciente reportó miocarditis aguda que debutó con falla cardíaca y fue reversible. En relación al COVID-19; Huang et $a l^{15}$ en 41 pacientes, observó daño miocárdico, expresado con incremento de troponina I ultrasensible con requerimiento de cuidado en algunos pacientes críticos y Wang et al. ${ }^{16}$ en una serie de 138 pacientes encuentran daño cardíaco agudo (7.2\%), choque (8.7\%), y arritmias (16.7\%). Con la evidencia escrita y la elevación enzimática cardíaca, el cuadro clínico de nuestra paciente se corresponde a una probable miocarditis viral con trastornos severos de la conducción, que explicaría la refractariedad a marcapasos transcutáneo, atropina y aminas vasoactivas.

\section{Conclusiones}

El presente caso clínico muestra una probable miocarditis viral asociada a SARS CoV-2, que genera síncope, bloqueo cardíaco avanzado, bradicardia extrema y paradas cardiorrespiratorias sucesivas, que preceden a sintomatología respiratoria. La importancia de éste reporte radica en informar a la comunidad médica presentaciones clínicas atípicas del COVID -19 que alerte la necesidad de ampliar la bioseguridad en la atención en etapa de pandemia.

\section{Referencias bibliográficas}

1. Wang, D. et al. Clinical characteristics of 138 hospitalized patients with 2019 novel coronavirus-infected pneumonia in Wuhan, China. JAMAhttps://doi.org/10.1001/jama.2020.1585 (2020). 
2. Huang, C. et al. Clinical features of patients infected with 2019 novel coronavirus in Wuhan, China. Lancet395, 497-506 (2020).

3. Turner, A. J., Hiscox, J. A. \& Hooper, N. M. ACE2: from vasopeptidase to SARS virus receptor. Trends Pharmacol. Sci.25, 291-294 (2004).

4. Alhogbani, T. Acute myocarditis associated with novel Middle East respiratory syndrome coronavirus. Ann. Saudi Med.36, 7880 (2016).

5. Wong, C. K. et al. Plasma inflammatory cytokines and chemokines in severe acute respiratory syndrome. Clin. Exp. Immunol.136, 95-103 (2004).

6. Sakabe, M., Yoshioka, R. \& Fujiki, A. Sick sinus syndrome induced by interferon and ribavirin therapy in a patient with chronic hepatitis C. J. Cardiol. Cases8, 173-175 (2013).

7. Nguyen JL, Yang W, Ito K, Matte TD, Shaman J, Kinney PL. Seasonal influenza infections and cardiovascular disease mortality. JAMA Cardiol 2016; 1: 274

8. Badawi A, Ryoo SG. Prevalence of comorbidities in the Middle East respiratory syndrome coronavirus (MERS-CoV): a systematic review and meta-analysis. Int J Infect Dis 2016; 49: 129 - 133.

9. Li SS, Cheng C, Fu C, Chan Y, Lee M, Chan JW, Yiu S. Left ventricular performance in patients with severe acute respiratory syndrome: a 30-day echocardiographic follow-up study. Circulation 2003; 108: 1798 - 1803.
10. Harris JE, Shah PJ, Korimilli V, Win H. Frequency of troponin elevations in patients with influenza infection during the 2017-2018 influenza season. Int J Cardiol Heart Vasc 2019; 22: 145 - 147.

11. Wit E D, Doremalen N. V, Falzarano D, Munster VJ. SARS and MERS: recent insights into emerging coronaviruses. Nat Rev Microbiol 2016; 14: $523-534$.

12. Yu C-M. Cardiovascular complications of severe acute respiratory syndrome. Postgrad Med J 2006; 82: 140-144.

13. Pan S, Zhang H, Li C, Wang C. [Cardiac arrest in severe acute respiratory syndrome: analysis of 15 cases]. Zhonghua Jie He He Hu Xi Za Zhi 2003; 26: $602-605$.

14. Alhogbani T. Acute myocarditis associated with novel Middle East respiratory syndrome coronavirus. Ann Saudi Med 2016; 36: 78 - 80

15. Huang C, Wang Y, Li X, Ren L, Zhao J, Hu Y, Zhang L, Fan G, Xu J, Gu X, Cheng Z, Yu T, Xia J, Wei Y, Wu W, Xie X, Yin W, Li H, Liu M, Xiao Y, Gao H, Guo L, Xie J, Wang G, Jiang R, Gao Z, Jin Q, Wang J, Cao B. Clinical features of patients infected with 2019 novel coronavirus in Wuhan, China. Lancet 2020; 395: $497-506$.

16. Wang D, Hu B, Hu C, Zhu F, Liu X, Zhang J, Wang B, Xiang H, Cheng Z, Xiong Y, Zhao Y, Li Y, Wang X, Peng Z. Clinical characteristics of 138 hospitalized patients with 2019 novel coronavirus-infected pneumonia in Wuhan, China. JAMA 2020; doi:10.1001/jama.2020.1585.

Received: 28 marzo 2020

Accepted: 5 mayo 2020 